\title{
Septal Lesions Impair the Acquisition of a Cued Place Navigation Task: Attentional or Memory Deficit?
}

\author{
C. Brandner and F. Schenk
}

Institut de Physiologie, Bugnon 7, CH-1005 Lausanne, Switzerland

These experiments were designed to analyze how medial septal lesions reducing the cholinergic innervation in the hippocampus might affect place learning. Rats with quisqualic lesions of the medial septal area (MS) were trained in a water maze and on a homing table where the escape position was located at a spatially fixed position and further indicated by a salient cue suspended above it. The lesioned rats were significantly impaired in reaching the cued escape platform during training. In addition rats, did not show any discrimination of the training sector during a probe trial in which no platform or cue was present. This impairment remained significant during further training in the absence of the cue. When the cued escape platform was located at an unpredictable spatial location, the MS-lesioned rats showed no deficit and spent more time under the cue than control rats during the probe trial. On the homing board, with a salient object in close proximity to the escape hole, the MS rats showed no deficit in escape latencies, although a significant reduction in spatial memory was observed. However, this was overcome by additional training in the absence of the cue. Under these conditions, rats with septal lesions were prone to devel op a pure guidance strategy, whereas normal rats combined a guidance strategy with a memory of the escape position relative to more distant landmarks. The presence of a salient cue appeared to decrease attention to environmental landmarks, thus reducing spatial memory. These data confirm the general hypothesis that MS lesions reduce the capacity to rely on a representation of the relation between several landmarks with different salience. @ 1998 Academic Press

\section{INTRODUCTION}

Chol inergic neurons located in the medial septum provide most of the cholinergic innervation of the hippocampal formation. F unctionally the septohippocampal cholinergic connection appears essential for the generation of the hippocampal theta rhythm (Alonso, Gaztelu, Bruno, \& Garcia-Austt, 1987; Vanderwolf, Kramis, Gillespie, \& Bland, 1975; Miller, Bickford, Wiser, \& Rose, 1995). Moreover, the activity of cholinergic fibers influences the development of long-term potentiation (LTP) in the hippocampus (Buszaky, 1992; Garcia \& J affard, 1992). The theta rhythm alters the firing rate of hippocampal pyramidal cells and facilitates long-term potentiation (Arai \& Lynch, 1992). This suggests that the activity of the hippocampus may be strongly affected by damage to the septohip-

We are grateful to Dr. G. Vantini for ChAT analyse and to M. Gafner for animal training assistance. Thanks are due to Elizabeth Mathew for careful reading of the manuscript. This research was supported by Grant No. 31.30068.90 from the Swiss National Science Fondation.

Correspondence should be addressed to C. Brandner, Institut de physiologie, Bugnon 7, 1005 Lausanne, Switzerland (e-mail: Catherine.Brandner@iphysiol.unil.ch). 
pocampal input. Medial septal lesions dramatically decrease the intrahippocampal metabolism of acetylcholine as measured by the activity of acetylcholine esterase (AChE) and choline acetyltransferase (ChAT) (Ginn \& Peterson, 1992; Gaspar, Heeringo, Markel, Luiten, \& Nyakas, 1992). The same lesions have been found to abolish the theta rhythm as well (Andersen, Bland, Myhrer, \& Schwartzkroin, 1979; Dutar, Bassant, Senut, \& Lamour, 1995).

Dysfunction of the hippocampus induced by lesions of the medial septum therefore may result in behavioral effects similar to those of hippocampal lesions and thus impair spatial abilities. Whereas an aspiration of the hippocampus has dramatic and permanent effects on the acquisition of place memory in a Morris navigation task (Morris, Garrud, Rawling, \& O'Keefe, 1982), selective lesions of the hippocampal formation have more subtle effects. F or example, a dramatic impairment in task acquisition is followed by a recovery of some subcomponents of the spatial performance if training is prolonged (see Schenk \& Morris, 1985; Morris, Schenk, Tweedie, \& J arrard, 1990) or conducted under particular conditions (Eichenbaum, Stewart, \& Morris, 1990; Whishaw, Cassel, \& J arrard, 1995). Significant residual spatial capacities are thus maintained following a selective destruction of a substructure of the hippocampal formation. Therefore, it is not clear which capacities ought to be preserved following a mere septohippocampal cholinergic loss.

Predicted by previous research, initial experiments showed that septal lesions impair the acquisition and maintenance of spatial memory in the Morris task (Hagan, Salamone, Simpson, I versen, \& Morris, 1988; Kelsey \& Laudry, 1988) but preserve the ability to solve nonspatial tasks (Gallagher \& Holland, 1992; Kelsey and Vargas, 1993). However, further studies revealed that place learning abilities in the Morris task were not systematically affected by septal lesions. A disconnection between preserved place learning abilities in the Morris navigation task and impaired performance in a radial maze task following lesions of the cholinergic forebrain system was reported (Decker, Radek, Majchrzak, \& Anderson, 1992; Sinden, Hodges, \& Gray, 1995).

This type of dissociation might be explained by the indirect role of the cholinergic system in spatial memory. Cholinergic activity appears to be mainly related to acquisition strategies (Whishaw, 1985; Whishaw, Mittelman, Bunch, \& Dunnett, 1987; Buhot, Soffie, \& Poucet, 1989; Scott J anis, Bishop, \& Dunbar, 1994) or to attention processes (Muir, Dunnett, Robbins, \& Everitt, 1992; Olton, Wenk, \& Markowska, 1991; Robbins, Everitt, Marston, Wilkinson, J ones, \& Page, 1989; Voytko, Olton, Richardson, Groman, Tobin, \& Price, 1994). It is thus likely that lesions of the cholinergic affect spatial learning processes but it is yet unclear whether this is due to: (1) an impairment in spatial memory per se, (2) a deficit in attentional processes, or (3) the reliance on less optimal strategies. The recent development of theoretical models of spatial memory processes (Poucet, 1993; O'Keefe, 1993; McNaughton, Knierim, \& Wilson, 1995) might allow a more analytical approach of the role of the septohippocampal cholinergic input in the expression of normal spatial abilities.

Typically, a cued spatial task is designed to demonstrate that rats with a specific deficit in spatial memory have preserved general attention, motoric abilities, and motivation (Whishaw \& Tomie, 1987). In general, the task is designed to enable normal rats to perform at a maximal level. However, we have found that a perinatal choline supplementation did enhance spatial mem- 
ory in intact rats when they were trained in the presence of a local cue (Schenk \& Brandner, 1995). This result suggests that spatial memory can be enhanced by an improved attention to rel evant local, as well as to more distant, contextual cues. It also suggests that normal rats combine a guidance and a spatial strategy for optimal performance.

The present study was designed to assess the effects of quisqualic lesions of the medial septal area (MS) on the acquisition and retention of two spatial tasks: the Morris navigation task and the homing hole board task. In the setup, the escape position was signal ed by a local cue placed in the immediate vicinity of the hidden goal. Experiments were aimed at analyzing the ability of MSlesioned rats to combine a rapid cue approach with a memory of the spatial position of the goal (hidden platform or escape hole). The requirement of a combined strategy is expected to induce a sel ective acquisition deficit in subjects with impaired spatial memory, as these rats are more prone to devel op a simple guidance strategy (Whishaw \& Petrie, 1988). In addition, probe trials conducted in the absence of the local cue and escape platform should reveal if rats with impaired attention to contextual cues are capable of goal location recall.

\section{EXPERIMENT 1}

This experiment was designed to assess the effects of medial septal lesions on the localization of a hidden platform signaled by a suspended cue. This study consisted of three phases: (1) A cue-fixed platform location phase, in which the cued escape platform remained at a fixed spatial location. (2) A no cue-fixed platform location phase, in which training was continued with the escape platform at the same location but in absence of the local cue. (3) A cuevariable platform location phase, in which the cue and the escape platform were randomly located in order to assess the animals' capability to rely on a local cue suspended above the hidden platform to reach the goal independently of its spatial position. A probe trial was conducted at the conclusion of training in the cue-fixed platform location phase and the no cue-fixed platform location phase in the absence of the platform and hanging cue to evaluate the spatial knowledge of the animals. During the cue-variable platform location phase, the cue and the escape platform were randomly located in order to assess the animals' capability to rely on a local cue suspended above the hidden platform to reach the goal independently of its spatial position. A probe trial in presence of the cue was conducted at the end of this phase to measure the attractivity of the cue itself.

Material and Methods

Subjects

Fifteen naive Long-Evans female rats bred and housed in our laboratory were tested in two behavioral tasks. Rat subjects were approximately 6 months old and weighed $230-260 \mathrm{~g}$ at the onset of experiment 1 . Rats were housed 3 to 5 per glass cage ( $40 \times 40 \mathrm{~cm}$ wide $\times 50 \mathrm{~cm}$ high). Food and water was provided ad libitum.

\section{Surgical Procedure}

Surgical procedures for medial septal lesions were performed under Nembutal anesthesia (55 mg/kg, ip). The rats were mounted in a stereotaxic apparatus 
(Kucera, 1970) with lambda and bregma horizontal. After exposure of the skull, holes were drilled and $0.5 \mu \mathrm{l}$ quisqualic acid (Tocris Neuramin; $0.12 \mathrm{M}$ solution in phosphate-buffered saline) was injected bilaterally into the medial septal area with a $0.2-\mathrm{mm}$ metal cannula for $5 \mathrm{~min}$. The following stereotaxic coordinates were used: $0.20 \mathrm{~mm}$ anterior to bregma, $\pm .25 \mathrm{~mm}$ lateral to the midline, $6.3 \mathrm{~mm}$ ventral to skull surface. Six rats recei ved quisqualic acid and five control animals received sham surgery, which consisted of lowering the empty cannula at the injection site. The skin was sutured, and the animals were allowed a 3week recovery period. Four animals served as nonoperated controls.

\section{Apparatus}

A large circular tank $(\varnothing 160 \mathrm{~cm}$, wall height $60 \mathrm{~cm})$ painted white filled with water $\left(25^{\circ} \mathrm{C}\right.$ ) made opaque by milk ( 0.5 liters) to a depth of $30 \mathrm{~cm}$ was used. The pool was positioned in a room containing several cues (door, posters, suspended dark cloth) at some distance from the pool wall (minimum, $100 \mathrm{~cm}$ ). Four orthogonal starting positions were situated around the perimeter of the pool, dividing its surface into four quadrants. An invisible escape platform made of transparent Plexiglas (diameter $9 \mathrm{~cm}$ ) could be placed in the center of each quadrant approximately $2 \mathrm{~cm}$ under the level of water. A black cylinder $(\varnothing 4.5 \mathrm{~cm}$, length $10 \mathrm{~cm}$ ) was suspended $30 \mathrm{~cm}$ above the platform for a cued procedure. A videocamera was placed directly above the center of the pool.

\section{Behavioral Procedure}

During the first phase, naive rats received 28 trials extended over 4 days (4, 8,8 , and 8 trials, respectively). The platform was at a fixed position for each rat. The black cue was suspended above the platform ("place and cue" condition). Four starting positions were pseudo-randomly distributed so that each position was used once in a block of four trials. In phase 1, a plastic grid starting platform fastened by two metal wires was suspended along the tank wall at one of four possible positions (Markowska, Long, J ohnson, \& Olton, 1993). At the beginning of each trial, the animal was placed on the above-mentioned platform at water level for $15 \mathrm{~s}$. The platform was then slowly lowered and removed, to initialize the trial. From trial 13 to the conclusion of phase 1 (trial 28) the traditional starting procedure used during the probe trial (placement into the water facing the wall of the pool) was alternated with the special one (placement on the plastic grid starting platform). The time required to locate the escape platform was recorded up to a maximum of $60 \mathrm{~s}$. In overtime, the rat was guided to the platform. At the end of each trial, the rat was allowed to remain on the escape platform for $15 \mathrm{~s}$ before being removed from the pool for the next trial (ITI about $15 \mathrm{~s})$. To measure remembrance of goal location, a 60-s probe trial (PT1) during which the rat was left in the pool without platform or cue was conducted on day 4, immediately following trial 24.

After a 6-week lapse, training was resumed with the escape platform at the same position excluding the suspended cue (retention trials, "place only" condition). Ten trials over a 2-day period (i.e., 5 and 5 trials, respectively) and a probe trial (PT2) following the 10th trial were conducted.

In the third phase ( 3 weeks later) training was resumed with the hanging cue and the platform placed randomly at one of four possible positions in the center of one of the quadrants in each trial. Twenty-eight trials extending over 
a 4-day period $(8,8,8$, and 4 trials, respectively) were conducted. The rats started at one of the four orthogonal starting positions. Different platform and starting positions were used during each trial, in a pseudo-random schedule, ensuring that each start and platform position was used twice within the eight daily trials. Moreover, the relative position of the starting and escape platform during each trial was arranged in order to avoid sequences of more than two identical (right or left) motoric responses. At the end of the fourth daily training session, all rats were given a special probetrial during which they were allowed to swim in the pool in the absence of the escape platform, but with the cue suspended in the center of a quadrant.

\section{Histology and Neurochemistry}

Upon completion of the behavioral experiments all the rats were sacrificed by decapitation. Microtome sections $(60 \mu \mathrm{m})$ were obtained from the anterior part of the brain, containing the septal nuclei, and stained with cresyl violet. The remainder of the brain was frozen and stored at $-80^{\circ} \mathrm{C}$ until the ChAT activity assays. After the training phase, the brains were dissected and weighed. For each brain, both left and right hippocampi were pooled and homogenized in 10 vol of $25 \mathrm{mM}$ sodium phosphate buffer, ph 7.4, using a sonicator (sonifier, B-30) equipped with a microtip. Homogenate was centrifuged at $10,000 \mathrm{rpm}$ for $60 \mathrm{~min}$ at $4^{\circ} \mathrm{C}$. The resulting supernatant fraction was used to determine the ChAT activity level.

ChAT activity was measured according to previously published procedures (Fonnum, 1975) with minor modifications. In particular, sample preparation and incubation conditions were performed according to Kaneda and Nagatsu (1985). The $\left[{ }^{14} \mathrm{C}\right]$ acetylcholine formed was isolated and measured according to the method of Schrier and Shuster (1967).

\section{Data Recording and Analysis}

During training in the water maze, each trial was videorecorded and the escape latency was measured. Recording of the escape latency started when the rat began to swim, regardless of whether the starting platform was used. For data analysis, ANOVAs were used. In reference to latency data, the standard deviation varied as the mean, so that a logarithmic transformation was implemented on the original data to stabilize the variance and avoid biases in the ANOVAs (Snedecor \& Cochran, 1967).

Four trials (block 5, trials 17-20) of the "place and cue" condition, five trials (block 2, trials 6-10) of the "place only" condition, as well as the probe trials were analyzed by an XY videotracker (Kukam S. A.). This provided the data measuring path length, number of crossings, and duration of time spent in the four sectors $(\varnothing 44 \mathrm{~cm})$ of the pool located in the center of each quadrant.

Results

\section{CueFixed Platform Location}

Training trials. The overall mean escape latencies were higher in the MS group than in the control group (Fig. 1). A two-way ANOVA of the escape latencies (logarithm of latencies) showed a significant difference between groups $(F(1,13)=19.42, p=.0007)$. A decrease in block repetitions $(F(6,78)$ 


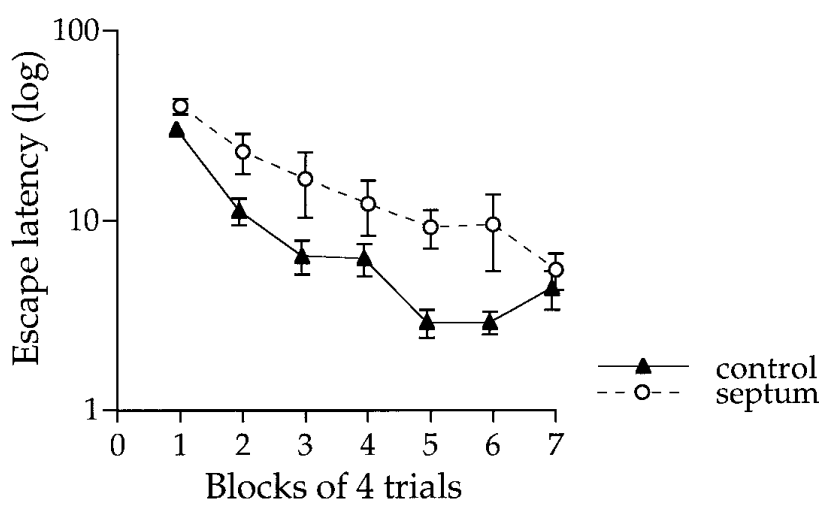

FIG. 1. Mean ( \pm SEM) escape latencies (logarithmic scale) during 4-day acquisition on the Morris navigation task in the presence of the hanging cue for MS-lesioned and for control rats.

$=33.21 ; \mathrm{p}=<.0001)$ without interaction between group and block $(\mathrm{F}(6,78)$ $=1.69$, ns) was also shown.

In control rats, the use of the starting platform significantly reduced the escape latencies. In contrast, this was not the case in MS-lesioned rats (Table 1). To analyze the escape strategies, two sections of the escape paths in block 5 (trials 17-20) were measured as follows: the first section equated the path distance between the starting point and the $40-\mathrm{cm}$ area around the escape platform, and the second section was the path distance from the first point crossing this 40-cm area to the platform ( $\mathrm{Fig}$. 2a). The length of the second path segment (in centimeters) was considerably longer in the lesioned rats (mean distance of the first segment: control $43.3 \pm 7.2$; MS lesions $62.2 \pm 9.8$; mean distance of the second segment: control $55.5 \pm 7.7$; MS lesions $198.2 \pm$ 65.6). A two-way ANOVA with the path segment as the repeated factor was conducted on the two path segments. It revealed a group effect $(F(1,13)=$ $17.08, p=.0012)$, an effect of repetition $(F(1,13)=8.97, p=.010)$, and an interaction between the above two factors $(F(1,13)=9.88, p=.008)$. There were no difference between groups on the mean distance of the first segment $(F(1,13)=1.31$, ns) but a lesion effect on the second segment $(F(1,13)=14.8$, $\mathrm{p}=.002$ ) was indicated upon subsequent analysis.

TABLE 1

\section{Effect of the Presence of the Starting Platform}

\begin{tabular}{|c|c|c|c|}
\hline & $\begin{array}{c}\text { Delayed start } \\
\text { (trials } 13,15,17 \\
19,22,24,25,27 \text { ) }\end{array}$ & $\begin{array}{l}\text { Immediate start } \\
\text { (trials } 14,16,18 \text {, } \\
20,21,23,26,28 \text { ) }\end{array}$ & $\begin{array}{l}\text { Start effect } \\
\quad(\log )\end{array}$ \\
\hline Control & 3.3 in. $( \pm 1.11)$ & 5.0 in. $( \pm 1.65)$ & $F(8,1)=48.77, p=.0001$ \\
\hline MS lesions & 8.2 in. $( \pm 5.05)$ & 10.7 in. $( \pm 7.08)$ & $\mathrm{F}(5,1)=1.23, \mathrm{~ns}$ \\
\hline $\begin{array}{l}\text { Group effect } \\
\text { (log) }\end{array}$ & $\begin{array}{c}F(1,13)=21.98 \\
p=<.0001\end{array}$ & $\begin{array}{c}F(1,13)=8.48 \\
p=.0043\end{array}$ & \\
\hline
\end{tabular}

Note. Mean latencies ( \pm SE ) of control and MS-Iesioned rats in trials with immediate or delayed start (i.e., the presence or the absence of the starting platform). One-way ANOVAs indicate condition or treatment effect. 
a

Sample of representative rats Training trial

Control

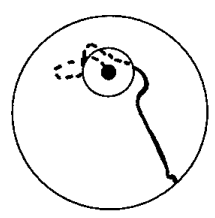

\section{Septal lesions}

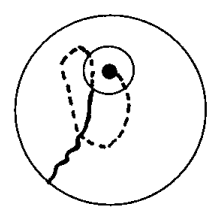

b Starting directional estimate Block 5

Control

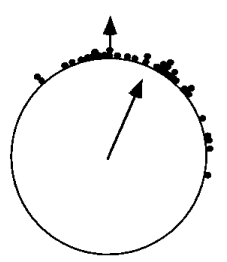

Septal lesions

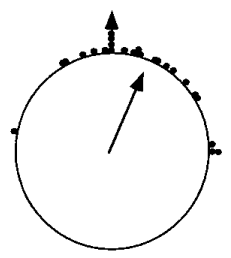

FIG. 2. (a) Two sections of the escape paths were measured. The first section (- - - - - ) is between the starting point and the crossing of an area around the escape platform (diameter of the platform: $14 \mathrm{~cm}$; diameter of the area: $42 \mathrm{~cm}$ ). The second section is the distance from this point until the rat reached the platform (- - - ). (b) The dots correspond to the orientation of each subject when leaving the start area on trials 17-20 (block 5). External arrow indicates platform direction. Inside arrow indicates vector length and direction (when all estimates are identical, agreement is total and $r=1$; when estimates are uniformly distributed throughout $360^{\circ}$, there is no agreement and $r=0$ ).

Start orientations were measured when the subject had moved $23 \mathrm{~cm}$ away from the edge of the pool. A one-way ANOVA conducted on the homeward component (corresponding to the length of the component of the mean individual vector in the home direction, Batschelet, 1981) showed that the starting direction of MS rats (Fig. 2b) did not vary from that of the control group ( $F(1$, $13)=.11, \mathrm{~ns})$.

Probetrial 1. During PT1, following trial 24, only control group rats showed a significant bias toward the training area. MS-lesioned rats did not focus on a particular sector (Fig. 3a).
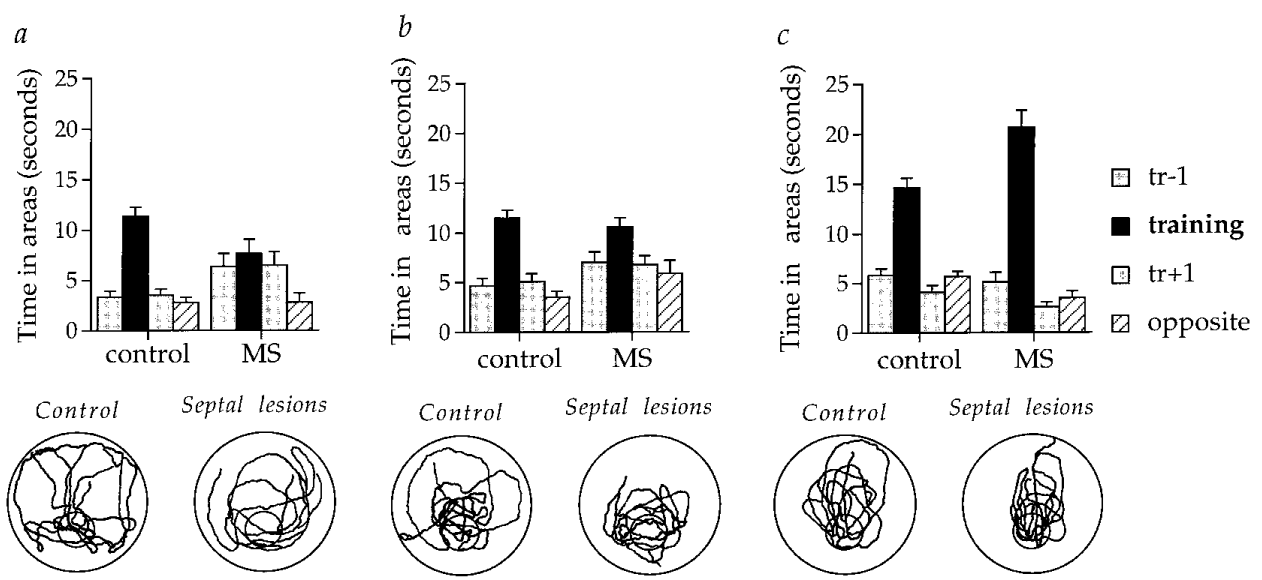

Training position (no cue)
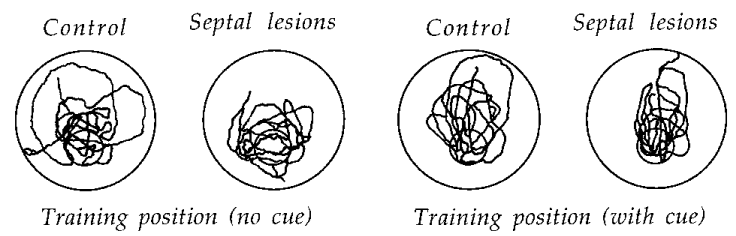

FIG. 3. Swimming paths by representative rats in each group and mean $( \pm S E M)$ of time spent in the four areas (diameter $42 \mathrm{~cm}$ around possible platform position) during 60-s probe trials. (a) Following training trials under the place and cue condition (PT1). (b) Following training under the place only condition (PT2). (c) Following training with the cued platform at variable positions (PT3). 


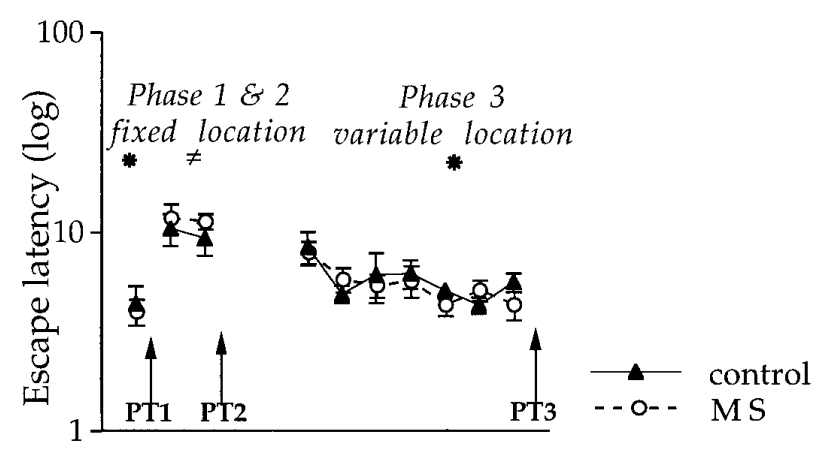

Blocks of 4 trials

FIG. 4. Mean ( \pm SEM) escape latencies (logarithmic scale) during the last block of training trials in the presence of the hanging cue $\left(^{*}\right)$ and the two blocks of training in absence of the cue $(\neq)$ in phase $1+2$. Mean $( \pm$ SEM) escape latencies (logarithmic scale) during training in phase 3 with the cued platform at variable positions.

A general analysis of variance with repetitions showed no group effect ( $F(1$, $13)=1.14, \mathrm{~ns})$, although a difference in the amount of time spent in the four sectors $(F(3,39)=27.67, p=<.0001)$ and a interaction between groups and sectors $(F(3,39)=7.42, p=.0005)$ were observed. An one-way ANOVA revealed that the control group spent a greater amount of time in the training sector than the MS group (treatment effect: $F(1,13)=6.70, p=.02$ ).

As shown in Fig. 3a, the lesioned rats presented stereotypic circular swimming patterns, whereas control rats swam directly toward the position where the platform was previously situated and concentrated their exploration around this area. A two-way ANOVA conducted on the number of crossings in the four sectors showed no group effect $(F(1,13)=3.58$, ns). A difference between sectors $(F(3,39)=17.10, p=<.0001)$ and a significant interaction between groups and sectors $(F(3,39)=4.07 ; p=.013)$ were seen. Further analyses indicated that MS rats intersected the area around the training spot as often as the control rats $(F(1,13)=1.43$, ns). However, MS rats failed to discriminate this particular area from the two adjacent areas (PLSD, $p=n s$ ) and visited the opposite sector less frequently (opposite vs all other sectors PLSD significant, with $p<.05$ ).

\section{No Cue-Fixed Platform Position}

Training trials. In the absence of the cue, the mean escape latency was not affected by MS lesions $(F(1,13)=2.46$, ns) and decreased throughout the two sessions $(F(9,117)=2.22, p=.02)$ without interaction (Fig. 4). Nevertheless, measuring of the mean swim distance (control: $218.02 \pm 65.9$; MS: 500.38 \pm 74.22 ) during the second block of five trials indicated that MS-lesioned rats covered significantly longer distances to reach the escape platform $(F(1,13)=$ 59.93, $\mathrm{p}=<.0001$ ) than control rats.

Probetrial 2. Despite an apparently good performance under the place only condition, a one-way ANOVA conducted on the percentage of time spent in the training area during PT2 revealed that MS rats spent less time than controls in the training area $(F(1,13)=5.27, p=.04)$. 
Figure $3 b$ shows that the lesioned rats did not develop normal searching in the training sector. Indeed, they entered the four sectors of the pool more often than did the control (control: $4.5 \pm 36$; MS: $5.8 \pm .37$ ). A two-way ANOVA with repetition conducted on the number of crossing in each sector revealed a group effect $(F(1,13)=11.66, p=.0046)$. A difference between sectors $(F(3$, $39)=10.8, p=<.0001$ ) without significant interaction between groups and sectors was seen.

\section{CueVariable Platform Location}

Training trials. No lesion effect was detected during this second phase of training (Fig. 4). A two-way ANOVA conducted on the mean escape latency showed that MS-lesioned rats were not impaired in their escape ability (group effect $F(1,13)=.12$, ns; block effect $F(6,78)=4.003, p=.002$ ).

Probe trial. During PT3, following trial 28, rats were allowed to patrol the pool in the presence of the suspended cue without an escape platform (Fig. 3c). A one-way ANOVA conducted on the percentage of time spent in the cued area revealed that MS rats concentrated their searching under the cue, similar to control rat behavior. It is to be noted, however, that MS rats did spend a greater amount of time in the cued sector than the control group $(F(1,13)=$ $11.72, \mathrm{p}=.0045)$.

\section{EXPERIMENT 2}

To assess the effect of a local cue in spatial learning on a solid surface and to eliminate a perfect control of approach trajectories to efficiently solve the navigation task, a large homing hole arena was used. In this task, rats were required to locate an escape hole situated at a fixed position and further signaled by a salient cue placed in its vicinity. In contrast with the Morris task, the presence of the covers and plugged holes during the probe trial only required the discrimination of the possible target position, thus reinforcing the searching capacity.

Material and Methods

Subjects

Rats from experiment 1 were used for this experiment. Animals were approximately 8 months old. Food distribution was limited to maintain $90 \%$ of ad lib. body weight.

\section{Apparatus}

The apparatus used for this experiment was a large circular homing board $(\varnothing 160 \mathrm{~cm})$ enclosed by $40-\mathrm{cm}$-high walls, as described elsewhere (Schenk, 1989). Fifteen possible escape holes were grouped in five triplets. Only one hole allowed passage to the subject's home cage, which was situated under the table. All holes were covered by a disk of white plastic identical to material covering the board. The room was rich in distal cues, and the table was indirectly lit by four $60-\mathrm{W}$ bulbs. A camera was placed directly above the center of the table. 


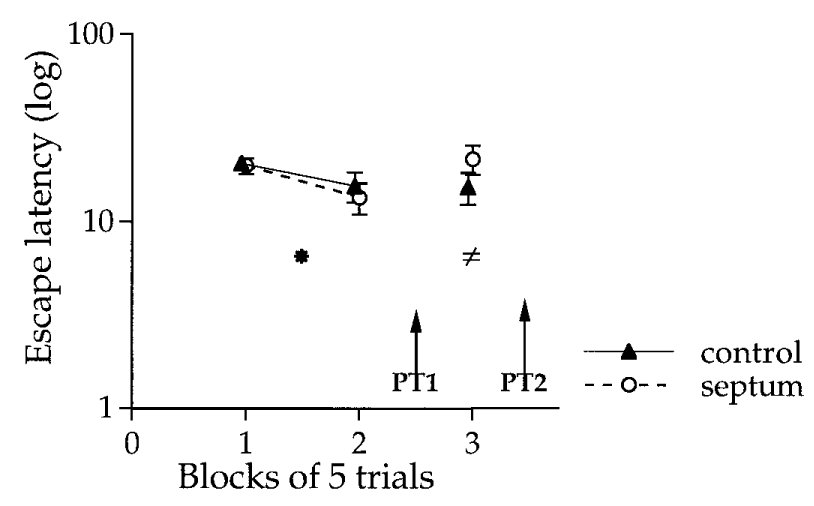

FIG. 5. Mean ( \pm SEM) escape latencies during training in the homing hole board for MSlesioned and control groups. The local cue was present in the center of the training sector during sessions $1(*)$ and 2 and absent during session $3(\neq)$.

\section{Behavioral Procedure}

The rat was deposited at one of five possible starting locations, facing the center of the arena. For the first three trials the subject was allowed up to $3 \mathrm{~min}$ to find and enter the escape hole. Rats which did not find the connected hole in the allotted time were gently guided toward the escape hole. Initially, the holes remained partially uncovered and were progressively masked during subsequent trials. The table was rotated after each trial, and the cover and the metal fitting of the hole through which the rat had entered were replaced, so that no relevant olfactory trace might remain attached to the training position.

All rats were trained to find the escape hole at a fixed location according to distant room cues. Each rat received two daily sessions of five trials (trials 110), with a salient cue placed in the middle of the triplet enclosing the escape hole. This cue was absent during the third daily training session (trials 1115). Two 72-s probe trials with no hole connected and no proximal cue present were conducted at the end of the second and third daily session.

\section{Data Analysis}

Each trial was videotaped and the escape latency was measured. As carried out for latency data obtained in the experiment 1, a logarithm transformation was operated on the original data to avoid biases in the ANOVAs (Snedecor \& Cochran, 1967). An XY videotracker (Kukam S. A.) was used to record a possible difference between groups in the distance covering five trials (session 3, trials 11-15) of the place only condition. Probe trials were also recorded and analyzed to collect data regarding path length, number of crossings, and duration time spent in different sectors of the arena.

\section{Results}

\section{Training Phase}

During the standard trials with the salient cue in the training triplet (place and cue condition), the performance of the lesioned rats did not differ from that of the control rats (Fig. 5). No effect of lesions was evident during this 
$a$
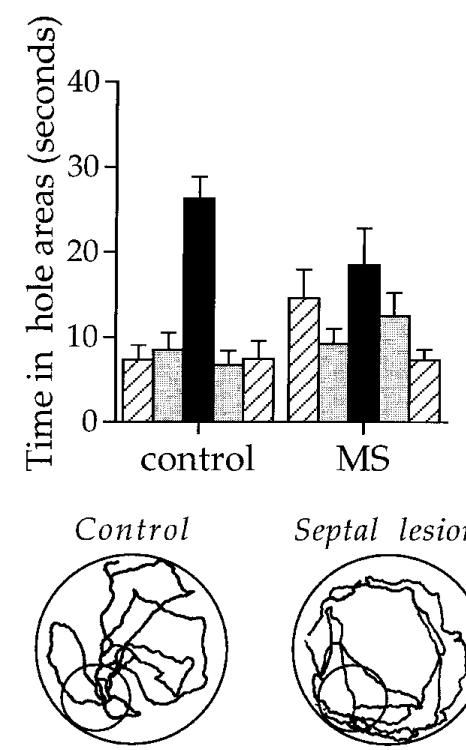

Training position (no cue) $b$

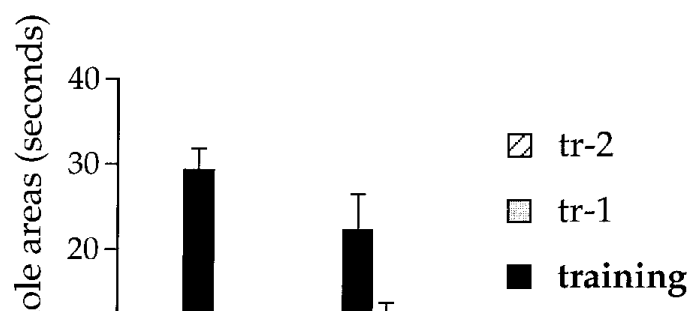

$\operatorname{tr}+1$

乙 $\operatorname{tr}+2$

FIG. 6. Paths by representative rats in each group and mean $( \pm S E M)$ of time spent in the five hole areas (diameter $42 \mathrm{~cm}$ ) during 72-s probe trials with no connected hole and no cue present, following training trials (a) under the place and cue condition (PT1) and (b) under the place only condition (PT2).

phase (group effect: $F(1,13)=.079, \mathrm{~ns})$ and the time spent before removing the plastic cover decreased in both groups (sessions effect: $F(1,13)=16.94$, $\mathrm{p}=.001$ ).

During session 3 (place only condition), the removal of the proximal cue increased the mean escape latency of the MS-lesioned rats (session 2: $13.4 \pm$ 2.5; session 3: $21.5 \pm 3.8$ ) but no such increase resulted in the control rats (session 2: $15.4 \pm 2.8$, session 3: $15.2 \pm 2.9$ ). This difference did not reach significance due to a high variance (group effect: $(F(1,13)=2.17$, ns). However, the analysis of the distance in the session 3 revealed that the MS-lesioned paths were significantly longer than those in the control group $(F(1,13)=$ $10.2, \mathrm{p}=.007)$.

\section{Probe Trials}

Figure 6a shows the amount of time spent in the five sectors of the arena during the first probe trial conducted at the end of the second daily session. A two-way ANOVA with repetition on the sectors indicated an effect of groups $(F(1,13)=5.89, p=.03)$, an effect of sectors $(F(4,52)=12.22, p=<.0001)$, and an interaction between these two factors close to the significance $(F(4,52)$ $=2.49, \mathrm{p}=.054)$. Further analyses indicated that only the control group discriminated the training sector from the other (control rats: $F(8,32)=14.28$, $\mathrm{p}=<.0001$, training versus all other sectors PLSD significant, with $\mathrm{p}<.05$; MS rats: $F(5,20)=1.88, p=n s)$. 
As in experiment 1, lesioned rats presented more stereotypic circular patterns of movement than the control rats and concentrated their exploration around thetraining area to a lesser degree. A two-way ANOVA conducted on the number of crossing in the five sectors indicated a group effect $(F(1,13)=5.21, p=.039)$ and an effect of repetition on the sectors $(F(4,52)=6.94, p=.0001)$ without interaction. Further analyses showed that if lesioned rats intersected the area around the training sector as frequently as the control rats $(F(1,13)=.21, p=$ ns), lesioned rats selectively failed to discriminate this area (Fisher's PLS $=n s$ ).

In contrast, MS rats showed a significant discrimination of the training sector during the second probe trial conducted at the end of the third daily session (Fig. 6b). A two-way ANOVA with repetition indicated no group effect $(F(1,13)=.97, p=n s)$ and an effect of sectors $(F(4,52)=22.71, p=<.0001)$, with no interaction between these two factors.

A two-way ANOVA conducted on the number of crossings of the second probe trial showed no difference between groups $(F(1,12)=3.75, p=n s)$ but a difference between sectors $(F(4,48)=10.53, p=<.0001)$ without interaction between these factors was seen. Further analyses confirmed that during the second probe trial conducted at the end of the training session under a place only condition, MS rats discriminated the training sector (training vs other sectors PLSD all significant, with $p<.05$ ).

\section{Neurochemistry}

Histological examination revealed bilateral damage to the medial septum in all subjects. ChAT activity expressed as micromoles per hour per $100 \mathrm{mg}$ of protein (mean \pm SEM ) in the hippocampus in rats with medial septal lesions and control was reduced by approximately $50 \%$ in the hippocampi of rats receiving an intraseptal quisqualic injection. One-way ANOVA conducted on ChAT activity expressed as micromoles per hour per $100 \mathrm{mg}$ of protein confirmed a marked decrease of enzymatic activity in hippocampi of lesioned rats $(F(1,9)=191.32, p=<.0001)$.

\section{GENERAL DISCUSSION}

Our results show that medial septal lesions, reducing ChAT activity in the hippocampus by $50 \%$, affected the performance in two place learning tasks conducted in the water or on a solid surface. The deficit was qualitatively similar in the two tasks, although to a lesser degree in the homing task.

In the Morris task, the MS-lesioned rats showed a reduction in escape performance regardless of the presence of a salient cue suspended above the platform. Although accurately oriented toward the platform when leaving the starting area, the lesioned rats often missed their target by a few centimeters and then proceeded with an extended correction loop before reaching the escape platform. Control rats also missed the target, but made an earlier and more efficient correction. If no cue and no platform were present, the lesion-induced escape deficit was coupled with a lack of spatial bias toward the training platform area and with a stereotypic swimming pattern during the probetrials. The spatial deficit was significantly reduced, but not entirely compensated for by further training without the suspended cue. The lesioned rats tended to demonstrate short escape latencies. However, an increased path length was 
evident compared to the control group. In the nonspatial task, when the cue was present during the probe trial, the stereotypic circle swimming strategy was abandoned. This suggested that such behavior was not a specific effect of the lesion and that the lack of a search pattern could be related to the absence of the relevant stimulus in the training area.

In the homing task, on a solid surface, the spatial deficit was clearly marked during the probe trial, following the removal of the goal cue. An increased escape path length was also evident during further training without the goal cue. In contrast to the Morris task, the lesioned rats were not impaired during escape when thegoal cue was in the center of thehole triplet with the connected hole. It might be that auxiliary local cues, such as the connected hole with possible olfactory cues from the connecting tube, allowed an easy discrimination of the target hole. The indication that lesioned rats relied on an auxiliary local cue to a greater extent than control rats is illustrated by the absence of a spatial bias in PT1 and an increased escape latency in session 3, following removal of the cue. In this task, however, lesioned rats appeared to recover a nearly normal discrimination of the training sector in PT2, subsequent to a few noncued training trials. The between-task difference might be related to the lower accuracy requirement of the homing task due to the presence of a perceptible hole (but blocked) in the training position during the probe trials. In the Morris task, the extended training period with the suspended cue might also have consolidated a cue approach strategy and stereotyped circular swimming action to the detriment of a memory of the platform position relative to that of distant environmental landmarks.

It is known that a salient local cue facilitates escape. It is assumed that a cued task does not require spatial memory per se, but rather an association between the cue and the goal for the development of a guidance strategy. It has also been demonstrated that lesions of the striatum affect the cued task, while lesions of the fornix reduce performance in place tasks (McDonald \& White, 1993; Devan, Goad, \& Petri, 1996). In most cued tasks, the platform is visible, so that a rapid escape can be based on the single rule of approaching the conspicuous platform. In our task, however, the cue does not precisely coincide with the platform and offers only a partial support to landing. When the rat is in the proximity of the target, the cue is positioned above its head and thus might appear less salient. An optimal performance in this situation must thus rely on a mixed strategy, i.e., a cue guidance combined with a memory of the platform position relative to the more distant room cues. The lesioned rats behaved as if they had developed stronger cue guidances and reduced attention to more distant spatial cues.

\section{Effects of Medial Septal Lesions in the Morris Navigation and the Radial Maze Tasks}

In general, performance in spatial tasks is reported to be affected by septal lesions, but these effects appear less evident in the M orris task than in a radial maze task. In the M orris task, some authors have reported that rats with septal lesions are impaired (Miyamoto, Kato, Narumi, \& Nagaoka, 1987; Hagan et al., 1988; Riekkinen, Aaltonen, Sirvio, \& Riekkinen, 1991). Other studies, however (Barones, Nanry, Mundy, Mcginty, \& Tilson, 1991; Boitano, Dokla, Parker, Stalzer, Norelli, \& Fiorini, 1990; Decker et al., 1992), have failed to observe 
any spatial deficit in place learning with the invisible platform. These discrepancies might be due in part to the lack of selectivity of the different types of septal lesions. F or example, el ectrolytic lesions impair the acquisition of spatial discrimination between two visible platforms in the Morris task, whereas lesions with quisqualic acid have no effect (Decker et al., 1992). I ntracerebroventricular administration of 192 IgG-saporin led to the disruption of place discrimination (Nilsson, Leanza, Rosenblad, Lappi, Wiley, \& Björklund, 1992) but intraseptal microinjections resulted in a marginal impairment in the Morris navigation task. A slight delay in task acquisition was reported by BergerSweeney, Heckers, Mesulam, Lappi, and Sharma (1994), whereas in another study, the lesioned rats showed a normal acquisition and were only impaired in a match-to-place version of the task (Baxter, Bucci, Gorman, Wiley, \& Gallagher, 1995).

In the radial maze tasks, the deficit in spatial memory following septal lesions is more systematic (Decker et al., 1992; Scott J anis, Bishop, \& Dunbar, 1994; Shen, Barnes, Wenk, \& McNaughton, 1996). A significant deficit in the radial maze has been reported in rats with septal lesions in spite of a normal performance in the Morris task (Decker et al., 1992). Moreover, Shen et al. (1996) have shown that rats with a septal lesion make frequent reentries in already-visited arms but like control rats learn to avoid never-baited arms. Obviously, the deficit in spatial learning induced by septal lesions is task dependent. The question is thus whether this is due to an indirect effect of the lesion upon the development of spatial representation.

\section{Attention or Memory Deficits?}

Although the general effect of cholinergic blockade is to reduce learning and memory capacities, the question remains why and how these capacities are affected. Our results, as well as the deficits observed in most spatial tasks, could be due to the impairment of general attentional processes (Muir, Everitt \& Robbins, 1994). Septal lesions with AMPA have minor effects on the acquisition of the Morris task (McAlonan, Wilkinson, Robbins, \& Everitt, 1995), but alter the balance of the response to an aversive conditioned stimulus and to the contextual cues, suggesting that the attentional deficit consists of an abnormal attention to context stimuli. The inverse effects were obtained following lesions of the dorsal noradrenergic bundle (Selden, Cole, Everitt, \& Robbins, 1990), a system also supposed to be involved in attentional processes. The attentional hypothesis could therefore be further specified to account for the function of the cholinergic system in learning processes.

\section{Less of the Same or Really Different?}

It is not clear how impaired attention should affect place learning. Learning might simply appear slower though not qualitatively different (Sarter \& Bruno, 1997). However, there are convergent indications that rats with septal lesions show abnormal learning strategies and the impairment in patrolling the radial maze has been related to stereotyped arm chains (Scott J anis et al., 1994). Misadapted strategies have often been detected in the Morris navigation task following administration of anticholinergic drugs (Whishaw, 1985, 1989; Whishaw \& Tomie, 1987; Whishaw \& Petrie, 1988). Reliance on a praxis strategy as a preventive in the development of a spatial search strategy was described 
by Day and Schallert (1996). An experimental training procedure was used by these authors to prevent the development of a praxis strategy in the Morris task. During the early acquisition phase, the rats were guided to a large escape platform, which prevented circling along the pool wall. The size of the training platform was then progressively decreased throughout training. Under this condition, escape latencies and distances, as well as the percentage of time spent in the training position, were apparently normal in rats treated with atropine. Similar to control rats, atropine-treated rats did not show any abnormal perseveration in the old training quadrant during the reversal phase. Unfortunately, however, there are no specific indications that the treated rats had memorized the new platform position like control rats. A rapid transfer to a new position in the same environment is expected from subjects showing a normal performance. Therefore it is not possible to conclude that cholinergic blockade allows for development of completely normal spatial abilities when inadequate strategies are suppressed by special training conditions.

The strategies released by the cholinergic deficit might be more successful in the Morris task than in the radial maze. Different strategies might lead to nearly normal performance due to an easier adaptation in a place learning task, as discussed by McAlonan et al. (1995). For example, Van Rijzingen, Gispen, and Spruijt (1997) have shown that rats maintained in enriched environments after fimbria fornix lesions are less impaired in the Morris task than those maintained in standard cages. This can be explained by a learning process of avoiding the wall of the pool and remaining for an extended duration in the half of the pool where the platform was situated. Rats with fornix lesions were also capable of learning the position of the submerged platform following training under special conditions, i.e., a single start (E ichenbaum et al., 1990) or alternate escape trials onto a visible and an invisible platform (Whishaw et al., 1995). In the classical version of the Morris task, the position of the escape platform remains stable relative to the environmental landmarks, so that similar approach paths or routes can be reinforced in successive trials. The cues around the pool differ by laboratory and are often not controlled. They might allow for the devel opment of a snapshot memory of the locale view around the platform, combined with a guidance to reach the training quadrant. A greater dependence on some salient cues might explain why rats with septal lesions are particularly poor in learning to escape in a quadrant with "minimal cue access" (Hodges, 1996) and why they are more easily disturbed by the removal of some salient cues (Eichenbaum et al., 1990).

\section{Spatial Learning Based on “Adapted Egocentric Strategies"?}

The question, however, is whether and how a strategy not based on allocentric spatial processing might account for the described recovery in the homing task or for the apparently normal acquisition of placememory shown by other authors (Decker et al., 1992). At least theoretically, a very simple model using an approach of three main cues might account for a quasi-normal performance in the Morris task (Wilkie \& Palfrey, 1987). However, it is unlikely that this model implies rapid transfer capacities since the approach of a new set of three cues might take the same amount of time as the original acquisition. Normal rats learn a new position in few trials. Rats with fornix lesions showing "normal" place memory are impaired in learning a new position in the same pool (Whis- 
haw et al., 1995). A similarly limited performance was observed in rats with selective lesions of the hippocampus (Morris et al., 1990). A particular strategy might allows for learning of a single position but not for encoding trial-specific information. Hence, the more systematic deficit reported in radial maze tasks. In this later task, the memory of which arms have al ready been visited in a given trial must require a more cognitive type of spatial representation.

An important property of an allocentric spatial representation supposes the integration of the relations between different landmarks or cues, by allowing to accurate orientation in spite of the removal of one of the salient cues. More specifically, it allows for place recognition in the absence of a specific local view (Schenk, Grobéty, \& Gafner, 1997). In the experiments reported above, the MS-lesioned rats showed an abnormal dependence on the suspended cue to reach the escape platform, whereas normal rats of the Long- Evans strain show a similar discrimination of the training sector, whether they had been trained with or without a cue hanging above the platform (Brandner \& Schenk, 1995). Actually, normal rats are ableto combinea cue guidance with an allocentric use of the distant landmarks. This capacity is enhanced in rats of the PVG strain treated with choline, who were more efficient at reaching the cued platform and had more accurately focused search behavior on the training position in the absence of the marking cue (Schenk \& Brandner, 1995). As in rats with septal lesions, this capacity to combine strategies is reduced in immature rats up to the fifth postnatal week (Schenk, 1985) and in senescent rats (Brandner \& Schenk, 1996).

One can thus hypothesize that "pseudo-spatial" strategies show a greater dependence relative to some salient cues and poor reversal learning capacities. We suggest that these strategies are based on progressive visual adjustment to some salient cues (snapshot memory, Cartwright and Collett, 1987) as viewed from the platform, very much as in the model by Wilkie and Palfrey (1987). In contrast, processing the spatial relations between different cues for an allocentric coding requires attention to each of them, despite large inequalities in salience. This may be due to differences in size, differing contrasts with the background, and varying distances from the pool and platform. It might thus be that the salience of different cues must be buffered or somehow equalized for the extraction of spatial relations, a process hypothetically impaired when the cholinergic innervation of the hippocampus is severely reduced. In O'Keefe's model (1991), a geometric center (centroid) is computed by the mapping system from processing the spatial relations between different cues. In this model, the theta rhythm plays a critical role in providing a sinusoidal reference to measure phase angles. This rhythm is generally abolished or at least impaired by lesions of the septum (Dutar et al., 1995), which must thus reduce the capacity to integrate the relative position of different cues. This suggests that attention to cues having a low salience might be reduced as a consequence of an impairment in theta rhythm. In the absence of a spatial representation elaborated by the hippocampus, the most salient cues would be selected for a guidance strategy. The presence of a conspicuous cue associated with the goal might reduce the salience of more distant cues, thus explaining the difficulty in adjusting approach movement relative to extra-arena room cues. This speculative interpretation cannot be firmly validated by our data. However, it suggests that working with a cue-controlled environment in which the salience of the cues might be quantified should allow for a better 
understanding of the brain processes underlying spatial performance as proposed by Sarter and Bruno (1997).

In conclusion, our results, together with this short review of an abundant literature, indicate that experiments conducted in the classical design of the Morris task cannot lead to a clear understanding of why and how various types of functional alterations of the hippocampal formation do affect spatial memory. More elaborate models as to the relations between spatial information processing by the brain and behavioral strategies should help in designing critical experiments.

\section{REFERENCES}

Alonso, A., Gaztelu, J. M., Bruno, W., \& Garcia-Austt, E. (1987). Cross-correlation analysis of septohippocampal neurons during theta-rhythm. Brain Research, 413, 135- 146.

Andersen, P., Bland, H. B., Myhrer, T., \& Schwartzkroin, P. A. (1979). Septo-hippocampal pathway necessary for dentate theta production. Brain Research, 165(1), 13-22.

Arai, A., \& Lynch, G. (1992). Factors regulating the magnitude of long-term potentiation induced by theta pattern stimulation. Brain Research, 598, 173- 184.

Barones, S., J r., Nanry, K. P., Mundy, W. R., McGinty, J . F., \& Tilson, H. A. (1991). Spatial learning deficits are not solely due to cholinergic deficits following medial septal lesions with colchicine. Psychobiology, 19, 41-50.

Batschelet. (1981). Circular statistics in biology. London: Academic Press.

Baxter, M. G., Bucci, D. J ., Gorman, L. K., Wiley, R. G., \& Gallagher, M. (1995). Selective immunotoxic lesions of basal forebrain cholinergic cells: Effects on learning and memory in rats. Behavioral Neuroscience, 109(4), 714- 722.

Berger-Sweeney, J ., Heckers, S., Mesulam, M. M., Lappi, D. A., \& Sharma, M. (1994). Differencial effects on spatial navigation of immunotoxin-induced cholinergic lesions of the medial septal area and nucleus basalis magnocellularis. The J ournal of Neuroscience 14(7), 4507-4519.

Blokland, A. (1996). Acetylcholine: A neurotransmitter for learning and memory? Brain Research Reviews, 21, 285- 300.

Boitano, J . J ., Dokla, C. P. J ., Parker, S., Stalzer, K., Norelli, N., \& Fiorini, M. (1990). Effects of medial septal lesions on activity and water maze performance. Society for Neuroscience Abstracts 16, 1248.

Brandner, C., \& Schenk, F. (1995). Is the cholinergic system involved in adjusting a balance between focused and selective attention? European J ournal of Neuroscience, 8, 81. [Abstract 33.13]

Brandner, C., \& Schenk, F. (1996). Effect of gender difference upon spatial performance during aging. European J ournal of Neuroscience, 9, 150. [Abstract 83.23]

Buhot, M.-C., Soffie, M., \& Poucet, B. (1989). Scopolamine affects the cognitive processes involved in selective object exploration more than locomotor activity. Psychobiology 17, 40- 417.

Buszaki, G. (1992). Two-stage model of memory trace formation: A role for "noisy" brain states. Neuroscience, 3, 551-570.

Cartwright, B. A., \& Collett, T. S. (1987). Landmark maps for honeybees. Biological Cybernetics, 57, 85- 93.

Day, L. B., \& Schallert, T. (1996). Anticholinergic effects on acquisition of place learning in the Morris water task: Spatial mapping deficit or inability to inhibit nonplace strategies? Behavioral Neuroscience 110(5), 998-1005.

Decker, M. W., \& McGaugh, J . L. (1991). The role of interactions between the cholinergic system and other neuromodulatory systems in learning and memory. Synapse, 7, 151- 168.

Decker, M. W., Radek, R. J ., Majchrzak, M.J ., \& Anderson, D. J . (1992). Differential effects of medial septal lesions on spatial-memory tasks. Psychobiology, 20, 9- 17.

Devan, B. D., Goad, E. H., \& Petri, H. L. (1996). Dissociation of hippocampal and striatal contributions to spatial navigation in the water maze. Neurobiology of Learning and Memory, 66, 305- 323. 
Dunnett, S. B., Everitt, B. J ., \& Robbins, T. W. (1991). The basal forebrain-cortical cholinergic system: interpreting the functional consequences of excitotoxic lesions. Trends in Neurosciences, 14, 494- 501.

Dutar, P., Bassant, M.-H., Senut, M.-C., \& Lamour, Y. (1995). The septohippocampal pathway: Structure and function of a central cholinergic system. Physiological Reviews, 75(2), 393- 427.

Eichenbaum, H., Stewart, C., \& Morris, R. G. M. (1990). Hippocampal representation in place learning. J ournal of Neuroscience, 10, 3531- 3542.

Fibiger, H. C. (1991). Cholinergic mechanisms in learning, memory and dementia: A review of recent evidence. Trends in Neuroscience, 14, 220- 223.

Fonnum, F. (1975). A rapid radiochemical method for the determination of choline acetyltransferase. J ournal of Neurochemistry, 24, 407- 409.

Gallagher, M., \& Holland, P. C. (1992). Preserved configural learning and spatial learning impairment in rats with hippocampal damage. Hippocampus, 2(1), 81-88.

Garcia, R., \& J affard, R. (1992). The hippocampo-septal projection in mice: Iong-term potentiation in the lateral septum. NeuroReport, 3, 193- 196.

Gaspar, E., Heeringo, M., Markel, E., Luiten, P. G., \& Nyakas, C. (1992). Behavioral and biochemical effects of early postnatal cholinergic lesion in hippocampus. Brain Research Bulletin, 28(1), 65- 71.

Ginn, S. R., \& Peterson, G. M. (1992). Rate of septohippocampal neurons following intracerebroventricular injections of colchicine. Brain Research Bulletin, 29(5), 635-642.

Hagan, J . J ., Salamone, J . D., Simpson, J ., I versen, S. D., \& Morris, R. G. M. (1988). Place navigation is impaired by lesions of medial septum and diagonal band but not nucleus basalis magnocellularis. Behavioral Brain Research, 27, 9-20.

Hagan, J . J ., J ansen, J . H. M., \& Broekkamp, C. L. E. (1987). Blockade of spatial learning by the M1 muscarinic antagonist pirenzepine. Psychopharmacology, 93, 470-476.

Hodges, H. (1996). Maze procedures: The radial-arm and water maze compared. Cognitive Brain Research, 3, 167-181.

Inglis, F. M., \& Fibiger, H. C. (1995). Increases in hippocampal and frontal cortical acetylcholine release associated with presentation of sensory stimuli. Neuroscience, 66, 81-86.

Kaneda, N., \& Nagatsu, T. (1985). Highly sensitive assay for cholineacetyltransferase activity by high-performance liquid chromatography with electrochemical detection. J ournal of Chromatography, 341, 23-30.

Kelsey, J . E., \& Laudry, B. A. (1988). Medial septal lesion disrupt spatial mapping ability in rats. Behavioral Neuroscience, 102, 289-293.

Kelsey, J.E., \& Vargas, H. (1993). Medial septal lesions disrupt spatial, but not nonspatial, working memory in rats. Behavioral Neurosciences, 107(4), 565-574.

Kucera, P. (1970). A new stereotaxic instrument for brain operations in laboratory rodents. Brain Research, 17, 499- 505.

Markowska, A. L., Long, J . M., J ohnson, C. T., \& Olton, D. S. (1993). Variable-intervale probe test as a tool for repeated measurements of spatial memory in the water maze. Behavioral Neuroscience, 4, 627-632.

McAlonan, G. M., Wilkinson, L. S., Robbins, L. S., \& Everitt, B. J . (1995). The effects of AMPAinduced lesions of the septo-hippocampal cholinergic projections on aversive conditioning to explicit and contextual cues and spatial learning in the water maze. European J ournal of Neuroscience, 7, 281- 292.

McDonald, R. J ., \& White, N. M. (1993). A triple dissociation of memory systems: Hippocampus, amygdala, and dorsal striatum. Behavioral Neuroscience, 107, 3- 22.

McNaughton, B. L., Knierim, J . J ., \& Wilson, M. A. (1995). Vector encoding and the vestibular foundations of spatial cognition: Neurophysiological and computational mechanisms. In M. S. Gazzaniga (Ed.), The cognitive neurosciences (pp. 585- 595). Cambridge, Massachusetts: MIT Press.

Messer, W. S., J r., Stibbe, J .R., \& Bohnett, M. (1991). Involvement of the septohippocampal cholinergic system in representational memory. Brain Research, 564, 66- 72.

Miller, C. L., Bickford, P. C., Wiser, A. K., \& Rose, G. M. (1995). Long-term potentiation disrupts auditory gating in the rat hippocampus. J ournal of Neuroscience, 15(8), 5820-5830.

Miyamoto, M., Kato, J ., Narumi, S., \& Nagaoka, A. (1987). Characteristics of memory impairment 
following lesioning of the basal forebrain and medial septal nucleus in rats. Brain Research, 419, 19-31.

Morris, R. G. M., Schenk, F., Tweedie, F., \& J arrard, L. (1990). I botenate lesions of hippocampus and/or subiculum: dissociating components of allocentric spatial learning. European J ournal of Neuroscience, 2, 1016- 1029.

Morris, R. G. M., Garrud, P., Rawling, J . N. P., \& O'Keefe, J . (1982). Place navigation impaired in rats with hippocampal lesions. Nature (London), 297, 681- 683.

Muir, J . L., Dunnett, S. B., Robbins, T. W., \& Everitt, B. J . (1992). Attentional functions of the forebrain cholinergic systems: Effects of intraventricular hemicholinium, physostigmine, basal forebrain lesions and intra cortical grafts on a multiple-choice serial reaction time task. Experimental Brain Research, 89, 611-622.

Nilsson, O. G., Leanza, G., Rosenblad, C., Lappi, D. A., Wiley, R. G., \& Bjorklund, A. (1992). Spatial learning impairment in rats with selective immunolesion of the forebrain cholinergic system. NeuroReport, 3, 1005- 1008.

O'Keefe, J. (1993). Hippocampus, theta, and spatial memory. Current Opinion in Neurobiology, 3, 917-924.

O'Keefe, J . (1991). The hippocampal cognitive map and navigational strategies. In J. Paillard (Ed.), Brain and space (Vol. 16, pp. 273-295). Oxford Univ. Press.

O'Keefe, J ., \& Nadel, L. (1978). The hippocampus as a cognitive map. Oxford: Clarendon.

Olton, D. S., Wenk, G. L., \& Markowska, A. M. (1991). Basal forebrain, memory and attention. In R. T. Richardson (Ed.), Activation to acquisition: Functional aspect of the basal forebrain cholinergic system, (pp. 247-262). Boston: Birkhäuser.

Orsetti, M., Casamenti, F., \& Pepeu, G. (1996). Enhanced acetylcholine release in the hippocampus and cortex during acquisition of an operant behavior. Brain Research, 724, 89-96.

Poucet, B. (1993). Spatial cognitive maps in animals: New hypotheses on their structure and neural mechanisms. Psychological Review, 100(2), 001-0020.

Riekkinen, P., J r., Aaltonen, M., Sirvio, J ., \& Riekkinen, P. (1991). Tetrahydroaminoacridine alleviates medial septal lesion-induced and age-related spatial reference but not working memory deficits. Physiology and Behavior, 49, 1147- 1152.

Robbins, T. W., Everitt, B. J ., Marston, H. M., Wilkinson, J ., J ones, G. H., \& Page, K. J . (1989). Comparative effects of ibotenic acid- and quisqualic acid-induced lesions of the substantia innominata on attentional function in the rat: Further implications for the role of the cholinergic neurons of the nucleus basalis in cognitive processes. Behavioral Brain Research, 35, 221 240.

Sarter, M., \& Bruno, J . P. (1997). Cognitive functions of cortical acetycholine: Toward a unifying hypothesis. Brain Research Reviews, 23, 28-46.

Schenk, F. (1989). A homing procedure for studying spatial memory in immature and adult rodents. J ournal of Neuroscience Methods, 26, 249- 258.

Schenk, F. (1985). Development of place navigation in rats from weaning to puberty. Behavioral and Neural Biology, 43, 69-85.

Schenk, F., \& Morris, R. G. M. (1985). Dissociation between components of spatial memory in rats after recovery from the effects of retrohippocampal lesions. Experimental Brain Research, 58, 11- 28.

Schenk, F., Grobéty, M.-C., \& Gafner, M. (1997). Spatial learning by rats across visually disconnected environments. The Quarterly J ournal of Experimental Psychology 50B(1), 54- 78.

Schenk, F., \& Brandner, C. (1995). Indirect effects of peri- and postnatal choline treatment on place-learning abilities in rat. Psychobiology, 23(4), 302-313.

Schrier, B. K., \& Shuster, L. (1967). A simplified radiochemical assay for cholineacetyltransferase. J ournal of Neurochemistry, 14(10), 977-985.

Scott J anis, L., Bishop, T. W., \& Dunbar, G. L. (1994). Medial septal lesions in rats produce permanent deficits for strategy selection in a spatial memory task. Behavioral Neuroscience, 108(5), 892-898.

Selden, N. R. W., Cole, B. J ., Everitt, B. J ., \& Robbins, T. W. (1990). Damage to ceruleo-cortical noradrenergic projections impairs locally cued but enhances spatially cued water maze acquisition. Behavioral Brain Research, 39, 29-51. 
Shen, J ., Barnes, C. A., Wenk, G. L., \& McNaughton, B. L. (1996). Differential effects of selective immunotoxic lesions of medial septal cholinergic cells on spatial working and reference memory. Behavioral Neuroscience, 110, 1181-1186.

Sinden, J . D., Hodges, H., \& Gray, J . A. (1995). Neural transplant and recovery of cognitive function. Behavioral Brainscience, 18, 10-35.

Snedecor, G. W., \& Cochran, W. G. (1967). Statistical methods (pp. 329-330). Ames: I owa State Univ. Press.

Sutherland, R. J ., Whishaw, I. Q., \& Kolb, B. (1983). A behavioral analysis of spatial localization following electrolytic, kainate or colchicine induced damage to the hippocampal formation in the rat. Behavioral Brain Research, 7, 133- 153.

Toumane, A., Durkin, T., Marighetto, A., Galey, D., \& J affard, R. (1988). Differential hippocampal and cortical cholinergic activation during the acquisition, retention, reversal and extinction of a spatial discrimination in an 8-arm radial maze. Behavioral Brain Research, 30, 225234.

Vanderwolf, C. H., Kramis, R., Gillespie, L. A., \& Bland, B. H. (1975). Hippocampal rhythmical slow activity and neocortical low voltage fast activity: relations to behaviour. In R. L. I saacson \& K. H. Pribram (Eds.), The hippocampus, neurophysiology and behaviour (Vol. 2, pp. 101-128).

Van Rijzingen, I. M. S., Gispen, W. H., \& Spruijt, B. M. (1997). Postoperative environmental enrichment attenuates fimbria-fornix lesion-induced impairments in Morris maze performance. Neurobiology of Learning and Memory, 67, 21- 28.

Voytko, M. L., Olton, D. S., Richardson, R. T., Groman, L. K., Tobin, J . R., \& Price, D. L. (1994). Basal forebrain lesions in monkeys disrupt attention but not learning and memory. J ournal of Neuroscience, 14, 167- 186.

Whishaw, I. Q. (1985). Cholinergic receptor blockade in the rat impairs locale but not taxon strategies for place navigation in a swimming pool. Behavioral Neuroscience, 99, 979- 1005.

Whishaw, I. Q. (1989). Dissociating performance and learning deficits on spatial navigation tasks in rats subjected to cholinergic muscarinic blockade. Brain Research Bulletin, 23, 347- 358.

Whishaw, I. Q., Cassel, J . C., \& J arrard, L. (1995). Rats with fimbria-fornix lesions display a place response in a swimming pool: A dissociation between getting there and knowing where. J ournal of Neuroscience, 15, 5779-5788.

Whishaw, I. Q., \& Petrie, B. F. (1988). Cholinergic blockade in the rat impairs strategy selection but not learning and retention of nonspatial visual discrimination problems in a swimming pool. Behavioral Neuroscience, 120, 662-677.

Whishaw, I. Q., \& Tomie, J .-A. (1987). Cholinergic receptor blockade produces impairments in a sensorimotor subsystem for place navigation in the rat: Evidence from sensory, motor, and acquisition tests in a swimming pool. Behavioral Neuroscience, 101, 603-616.

Whishaw, I. Q., Mittelman, G., Bunch, S. T., \& Dunnett, S. B. (1987). I mpairments in the acquisition, retention and selection of spatial navigation strategies after medial caudate-putamen lesions in rats. Behavioural Brain Research, 24, 125- 138.

Wilkie, D. M., \& Palfrey, R. C. (1987). A computer stimulation model of rats' place navigation in the Morris water maze. Behavior Research Methods, Instruments and Computers, 19, 400403.

Worden, R. (1992). Navigation by fragment fitting: A theory of hippocampal function. Hippocampus, 2, 165- 188. 